\title{
A Clinico-Haematological Study of Causes of Pancytopenia
}

\author{
Arvind Chouhan¹, Fuzail Ahmad², Manisha Jain³
}

${ }^{1}$ Department of Medicine, GMC, Vidisha, Madhya Pradesh, India. ${ }^{2}$ Department of Pathology, GMC, Vidisha, Madhya Pradesh, India. ${ }^{3}$ Department of Obstetrics and Gynaecology, GMC, Vidisha, Madhya Pradesh, India.

\section{ABSTRACT}

\section{BACKGROUND}

This is a clinic-haematological study, conducted in Sidhanta Hospital, Bhopal, to identify the causes of pancytopenia. Out of total 1200 cases of complete hemograms, 56 cases were categorized as pancytopenias, and out of these, 24 cases were subjected to bone marrow examination. The remaining 32 cases of pancytopenia, were put on periodic follow up with peripheral blood smear examination, based on clinical data. Pancytopenia is the simultaneous presence of anaemia, leucopenia and thrombocytopenia that may result from various disease processes, involving the bone marrow primarily or secondarily. Pancytopenia is reduction in all the three cellular components of the blood, namely red blood cells (RBCs), leucocytes (WBCs) and platelets. It is a common entity which is encountered by practitioners. The presentation is in the form of cytopenias leading to infections, anaemia, or bleeding manifestations. All the cases of pancytopenia need a through approach to reach to the cause of the same so that it can be managed in the best possible manner. Cytopenias are reduction in any of the three cellular components of the blood i.e. RBCs, WBCs or platelets. It can be reduction in two cellular components (bicytopenia) or a reduction in all the three cellular components (pancytopenia). In bicytopenia, the most common combination to be seen is anaemia and thrombocytopenia, whereas the least common is leucopenia with thrombocytopenia. (1) For practical purposes, it should have haemoglobin $<10 \mathrm{~g} \%$, absolute neutrophil count $<1,500 /$ cumm and platelets < $1,00,000 /$ cumm. It is labelled as severe when the three values are $<7 \mathrm{~g} \%$, $<$ $500 /$ cumm and $<20,000 /$ cumm respectively. The purpose of this study was to find out different causes of pancytopenia and the use of bone marrow examination in evaluation of pancytopenia.

\section{METHODS}

This is a cross sectional study, conducted exclusively in the Department of Medicine, at a tertiary care hospital, in Bhopal from August 2019 to December 2019. A total of 56 cases of pancytopenia were analysed with clinico-haematological features. Criteria for diagnosis of pancytopenia were: Haemoglobin less than $10 \mathrm{gm} / \mathrm{dl}$, TLC less than $4000 / \mathrm{mm} 3$ and platelet count less than $1,00,000 / \mathrm{mm} 3$. We have correlated the complete hemogram findings with bone marrow examination (if required) and peripheral smear examination in order to analyse the root cause of every case of pancytopenia. Pancytopenia is a haematological entity, we have to analyse the cause of it in order to find out the correct diagnosis and treat the patient accordingly. Bone marrow examination is useful in the investigation of PUO (pyrexia of unknown origin), as it leads to an etiological diagnosis in many of the cases.

\section{RESULTS}

In these 56 cases, only 24 cases (42.85\%) were subjected for bone marrow examination. Commonest cause of pancytopenia was episode of viral fever constituting 28 cases (50\%).

\section{CONCLUSIONS}

In cases of PUO, bone marrow examination is a very useful investigation. In cases diagnosed as idiopathic thrombocytopenic purpura (ITP), when the patient does not show improvement in counts, a repeat bone marrow examination should be done, as very rarely; acquired amegakaryocytic thrombocytopenia may be the cause. Though bone marrow examination is an absolute indication in cases of pancytopenia, it is important to wait for at least $2-3$ weeks, and do a repeat hemogram, especially in cases of viral fever where the counts usually improve after fever subsides.
Corresponding Author: Dr. Fuzail Ahmad, C-1/J, Chargmli, Bhopal-462016, Madhya Pradesh, India. E-mail: fuzail2008@rocketmail.com

DOI: $10.14260 / j e m d s / 2021 / 716$

How to Cite This Article:

Chouhan A, Ahmad F, Jain M. A clinicohaematological study of causes of pancytopenia. J Evolution Med Dent Sci 2021;10(40):3533-3537,

$10.14260 /$ jemds/2021/716

Submission 20-01-2021,

Peer Review 11-09-2021,

Acceptance 17-09-2021,

Published 04-10-2021.

Copyright (C) 2021 Fuzail Ahmad et al. This is an open access article distributed under Creative Commons Attribution License [Attribution 4.0 International (CC BY 4.0)]

\section{KEY WORDS}

Pancytopenia, Bone Marrow, Viral Fever 


\section{BACKGROUND}

Pancytopenia is a challenging clinical entity for clinicians. Pancytopenia is reduction in all the three cellular components of the blood, namely red blood cells (RBCs), leucocytes (WBCs) and platelets. The underlying pathology and severity of symptoms determine the management strategy. Laboratory studies play a central role in determining the aetiology of pancytopenia and deciding the proper management. A wide spectrum of primary and secondary disorders affects the bone marrow and may manifest as pancytopenia. Pancytopenia could present with hypocellular normocellular and hypercellular bone marrow. Causes of pancytopenia may range from infectious to many non-infectious causes primary or secondary. ${ }^{1}$ Pancytopenia is a common entity which is encountered by practitioners. The presentation is in the form of cytopenias leading to infections, anaemia or bleeding manifestations. All the cases of pancytopenia need a through approach to reach to the cause of the same so that it can be managed in the best possible manner. This paper will mainly concentrate on the approach to a case of pancytopenia. Cytopenias are reduction in any of the three cellular components of the blood i.e. RBCs, WBCs or platelets. It can be reduction in two cellular components (bicytopenia) or a reduction in all the three cellular components (pancytopenia). In bicytopenia, the most common combination to be seen is anaemia and thrombocytopenia, whereas the least common is leucopenia with thrombocytopenia platelets $<1,00,000 /$ cumm. It is labelled as severe when the three values are $<7 \mathrm{~g} \%,<500 /$ cumm and $<20,000 /$ cumm respectively.

The complete haematological work up including a good peripheral blood smear examination, bone marrow aspiration and biopsy with clinical correlation is of utmost importance to evaluate the cause of pancytopenia and planning further investigations and treatment. ${ }^{2}$

Pancytopenia basically arises secondarily to four main aetiologies i.e. bone marrow failure, marrow space occupying lesions, effective production by marrow or peripheral destruction of hematopoietic cells. ${ }^{3}$ The causes can be both congenital and acquired. The inherited causes of bone marrow and their salient features are summarized in Table 1.

\begin{tabular}{|c|c|c|}
\hline Disease & Defect & Features \\
\hline Fanconi anaemia & $\begin{array}{c}\text { Defect in DNA repair leads to } \\
\text { increased chromosomal } \\
\text { breakage with cross linking } \\
\text { agents) }\end{array}$ & $\begin{array}{l}\text { Skeletal anomalies (skeletal, } \\
\text { thumb), short stature, } \\
\text { urogenital anomalies }\end{array}$ \\
\hline $\begin{array}{l}\text { Dyskeratosis } \\
\text { congenita }\end{array}$ & Shortened telomeres & $\begin{array}{c}\text { Triad (leukoplakia, nail } \\
\text { dystrophy, lacy skin } \\
\text { pigmentation), pulmonary } \\
\text { fibrosis }\end{array}$ \\
\hline $\begin{array}{c}\text { Shwachman diamond } \\
\text { syndrome }\end{array}$ & Mutation in SBDS gene & $\begin{array}{l}\text { Exocrine pancreatic } \\
\text { insufficiency }\end{array}$ \\
\hline $\begin{array}{c}\text { Congenital } \\
\text { amegakaryocytic } \\
\text { thrombocytopenia }\end{array}$ & $\begin{array}{c}\text { Myeloproliferative Leukemia } \\
\text { Virus Oncogene }\end{array}$ & $\begin{array}{l}\text { Severe thrombocytopenia with } \\
\text { its sequelae }\end{array}$ \\
\hline $\begin{array}{l}\text { Hemophagocytic } \\
\text { lymphohistiocytosis }\end{array}$ & Multiple genes PFR1, UNC13D & $\begin{array}{c}\text { Fever, splenomegaly, hepatitis, } \\
\text { rash }\end{array}$ \\
\hline \multicolumn{3}{|c|}{ Table 1. Inherited Causes of Bone Marrow Failure } \\
\hline
\end{tabular}

The acquired causes of pancytopenia include noninherited aplastic anaemia, megaloblastic anaemia, malignant marrow infiltrative disorders (acute leukaemias, myelodysplastic syndromes, solid tumours), non-malignant infiltrative disorders (storage disorders, osteopetrosis), infections (HIV, tuberculosis, hepatitis viruses, EBV, CMV), immune disorders (lupus, Evan's syndrome, thymoma), acquired clonal bone marrow failure disorder (PNH), metabolic (anorexia nervosa) and peripheral destruction of blood cells i.e. hypersplenism. ${ }^{4}$

Pancytopenia is always to be approached in a systematic manner through proper history, examination and then moving on to laboratory investigations as the first two parts if done properly and a systematic manner will lead very near to the diagnosis. The history should include the salient points viz age (inherited causes of bone marrow failure), sex, duration of symptoms (tells about the severity), bone pains (acute leukaemias), fever (infections, acute leukaemias), night sweats (Hodgkin's disease), malaise, weight loss (tuberculosis, malignancy), bleeding from any site (magnitude of thrombocytopenia), jaundice (hepatitis viruses), joint pain, rash, photosensitivity (lupus), any radiation exposure, exposure to potentially toxic chemicals, treatment history including herbals and drug intake, blood transfusions, dietary history (megaloblastic anaemia, anorexia nervosa), occupational exposure history (benzene).

A thorough examination should be done including vitals, anthropometry, general physical examination and systemic examination. Some salient features in examination will point towards particular aetiologies like: anthropometry including stature (short stature in Fanconi anaemia) dysmorphic features Fanconi anaemia), pallor (severity of anaemia), icterus (hepatitis viruses), lymphadenopathy (leukaemias), oedema, sings of congestive heart failure (CHF), stomatitis, cheilitis (neutropenia, nutritional deficiency), triad of nail dystrophy, leukoplakia, skin pigmentation (dyskeratosis congenita), oral candidiasis, pharyngeal exudates (neutropenia), petechiae, purpura, hyperpigmentation, sternal tenderness (acute leukaemias), gum hypertrophy (acute myeloid leukemia), hepatosplenomegaly, joint swelling, sinusitis (lupus).

Moving on to the laboratory investigations of pancytopenia, it requires complete blood counts with RBC indices, reticulocyte count and a peripheral blood examination. Bone marrow examination: aspiration and biopsy (to know the cellularity of the marrow) and specific investigations to find the causes leading to pancytopenia are needed. Complete blood counts will show that all the 3 cell lines are decreased. In bone marrow failure where there is ineffective production of the cells by the marrow, the reticulocyte counts will be decreased, whereas it will be higher in cases where pancytopenia is secondary to the peripheral destruction of the cellular components of the blood. Aniso- poikilocytosis i.e. variation in the sizes of RBCs along with the change in their shapes is very prominent in myelofibrosis whereas it is invariably absent in acute leukaemias. Peripheral smears can show blasts (acute leukaemias, myelofibrosis, subleukaemic leukaemias) or plasmacytic cells (multiple myeloma). RBC and WBC precursors in the peripheral blood are not typical of aplastic anaemia, so their presence in pancytopenia suggests diagnosis other than aplastic anaemia. Howell- Jolly bodies (basophilic nuclear remnants) can be found in the peripheral smear of megaloblastic anaemia or myelodysplastic syndrome. Hypersplenism and myelodysplastic syndrome peripheral smear can show giant platelets whereas normal 
sized platelets are found in aplastic anaemia. The granulations of the neutrophils are to be taken into account; toxic granules are found in infections whereas hypo granulation of the neutrophils is a characteristic of myelodysplastic syndromes. The neutrophils can have hypersegmentation (megaloblastic anaemia) or hypo segmented (myelodysplastic syndromes, chronic leukaemias). Multiple myeloma and cases of infections on the other hand will show an increase in ESR (erythrocyte sedimentation rates).

Bone marrow examination is almost always indicated in cases of pancytopenia unless cause is very apparent. Both aspiration and biopsy of the bone.

\section{METHODS}

This is a cross sectional study, conducted exclusively in the Department of Medicine, at a tertiary care hospital in Bhopal from August 2019 to December 2019. A total of 56 cases of pancytopenia were analysed with clinico-haematological features. Criteria for diagnosis of pancytopenia were: Haemoglobin less than $10 \mathrm{gm} / \mathrm{dl}$, TLC less than $4000 / \mathrm{mm} 3$ and platelet count less than $1,00,000 / \mathrm{mm} 3 .^{3}$ We have correlated complete hemogram findings with bone marrow examination (if required) and peripheral smear examination in order to analyse the root cause of every case of pancytopenia. Pancytopenia is a haematological entity, we have to analyse the cause of it in order to find to the correct diagnosis and treat the patient accordingly. Bone marrow examination is useful in the investigation of PUO (pyrexia of unknown origin), as it leads to an etiological diagnosis in many of the cases.

Bone marrow aspiration smears and trephine biopsy sections, in patients, fulfilling the criteria of pancytopenia were examined. Bone marrow aspiration was performed using Salah needle either from posterior iliac crest or sternum and biopsy with Jamshidi needle from posterior iliac crest, under local anaesthesia, with informed consent.

The relevant clinico-haematological parameters were recorded. The causes of pancytopenia were analysed based on clinico-haematological parameters, including peripheral blood film, bone marrow aspiration, bone marrow biopsy (in cases of dry tap), clinical features, age, gender and compared with the various studies published in literature.

The bone marrow aspiration smears were stained with Leishman's stain and the trephine biopsy core was decalcified, routinely processed, embedded in paraffin and sections stained with haematoxylin and eosin. Our study presented with 56 cases of pancytopenia, over a period of 3 months. Our study has been exclusively been performed in a tertiary care hospital of Bhopal.

The period of study was during the winter months, with increased frequency of viral fevers. Hence, one should not be in a hurry to do a bone marrow examination, as the transient decrease in the counts, may improve, as the fever subsides and also it is important not to hesitate doing the procedure. All those patients who came to our centre during the time frame of the study and who fulfilled the inclusion criteria were included in the study.

\section{Statistical Analysis}

There has been noticed a phenomenal $100 \%$ correlation between bone marrow aspirate and bone marrow biopsy examination in all the 56 cases in which bone marrow examination was performed. The diagnosis of bone marrow aspirate completely matched up and correlated with diagnosis and findings of bone marrow biopsy.

\section{RESULTS}

\begin{tabular}{|cc|}
\hline No. of cases & Diagnosis on Bone Marrow Aspiration \\
6 & Megaloblastic anaemia \\
6 & Hypoplastic anaemia \\
2 & Amegakaryocytic thrombocytopenia \\
2 & ALL and lymphoma (2 each) \\
4 & Improvement with vitamin B12 \\
2 & Storage disease and myelofibrosis (2 each) \\
2 & Tubular granuloma \\
\hline \multicolumn{2}{|c|}{ Table 2. Findings in Bone Marrow Examination } \\
\hline
\end{tabular}

A total of 1200 complete hemograms were done during a period of 3 winter months at tertiary care hospital in Bhopal. Out of which 56 cases fulfilled the criteria of pancytopenia, in these 56 cases, only 24 cases (42.85\%) cases were subjected for bone marrow examination.

The male to female ratio in cases of pancytopenia was 1 : 3.5. The age of the patients ranged from $6-65$ years. The most common age group was $2^{\text {nd }}$ decade constituting $28.7 \%$ of the cases, followed by $4^{\text {th }}$ decade $(14.3 \%)$ and $1^{\text {st }}$ and $3^{\text {rd }}$ decade each of $10.7 \%$ and $4^{\text {th }}, 5^{\text {th }}$ and $6^{\text {th }}$ decade constituted each $7.1 \%$. In $57.15 \%$ (32 cases) of pancytopenia, bone marrow examination was note done, as the counts improved, when a repeat hemogram was done, after a period of 10 to 15 years. The case details based on aetiology will be discussed.

\section{DISCUSSION}

We have correlated the complete hemogram findings with bone marrow examination (if required) and peripheral smear examination in order to analyse the root cause of every case of pancytopenia. Pancytopenia is a haematological entity, we have to analyse the cause of it in order to find out the correct diagnosis and treat the patient accordingly. Bone marrow examination is useful in the investigation of PUO (pyrexia of unknown origin), as it leads to an etiological diagnosis in many of the cases.

In the spectrum of bone marrow changes observed, in the present study, one case of pancytopenia in a female aged 32 years, with PUO, the bone marrow aspiration was a dry tap. The biopsy sections showed hypocellular marrow with collections of lymphocytes and granulomas located in the interstitium. The granulomas were ill defined, with collections of epithelioid cells and foci with necrosis. Langhan's type of giant cells, were not observed. Erythropoiesis, megakaryopoiesis and granulopoiesis were suppressed. Granulomas in marrow aspiration smears are difficult to identify probably because of the fibrosis, in and around the granulomas. Granulomas in bone marrow are an uncommon findings in western studies ${ }^{5,3}$ but is much higher in studies from India. ${ }^{6}$ In the present study, morphology of the granulomas in bone marrow is similar to those seen in 
other organs. There are no distinguishing features in the morphology of bone marrow granulomas, to permit a definitive diagnosis of the underlying condition. ${ }^{6}$ Though the granulomas associated with caseous necrosis and Langhan's type giant cells would be more frequent in tuberculosis. The finding needs to be correlated with clinical, laboratory (culture, serology) and radiological data, to arrive at an etiological diagnosis. Bone marrow culture also plays a vital role, in identifying the aetiology, when blood culture is negative. In one study, 6 Brucella organisms were grown, in one case. In the present study, the case of granulomas in bone marrow biopsy, on follow up, after 1-month ATT (anti tuberculous treatment), showed improvement in blood cell counts. A rare case of acquired amegakaryocytic thrombocytopenia (AATP) was encountered in the present study, which was first treated as immune thrombocytopenia and later diagnosed as AATP. The patient was a female child 14 years of age, diagnosed as ITP, 3 years back. O/E purpuric spots were observed throughout the body and there was no organomegaly. Initially, 3 years back, the episode of thrombocytopenia occurred, after an episode of viral fever and diagnosed as ITP. Since then, the patient was on prednisolone, on \& off (for the last 3 years). Present episode of thrombocytopenia did not show any improvement in platelet counts, even after treatment with prednisolone. A bone marrow examination was done, as the peripheral blood film showed anaemia and leucopenia along with thrombocytopenia. Bone marrow aspiration smears showed hypercellular particles with erythroid hyperplasia and micronormoblastic type of erythropoiesis. Myelopoiesis showed orderly maturation. Megakaryopoiesis was markedly suppressed, with absence of megakaryocytes. Lymphocytes and plasma cells were within normal limits. There was no evidence of any deposit or leukemic process. Hence, a diagnosis of acquired amegakaryocytic thrombocytopenic purpura was made. In the management of the present case, a whole blood transfusion was given, prednisolone was stopped and planned to be treated with Antithymocyteglobulin (ATG) $15 \mathrm{mg} / \mathrm{kg}$. Several cases of amegakaryocytic thrombocytopenic purpura have been reported following prolonged administration of DES or other estrogenic hormones. Prednisolone in massive doses is moderately hemato suppressive and in some individuals prolonged administration of conventional doses $(60 \mathrm{mg} / \mathrm{dl})$ may depress platelet production, ironically this depression has been observed most often in patients with ITP who were continued on prednisolone for more than 2 weeks after platelet levels responded.

In the present study, 2 case of hypoplastic marrow was recorded in a male patient of age 63 years, with incidence of $10.7 \%$ aplastic anaemia. The incidence of aplastic anaemia varies from $10-52.7 \%$ of all pancytopenia patients and is the commonest cause of pancytopenia reported from various studies throughout the world. ${ }^{7}$ Bone marrow aspiration was dry tap in 4 cases and 2 case smears show fatty fragments with trapped lymphocytes and plasma cells. Bone marrow trephine biopsy in all 6 cases showed increased fat spaces and decreased cellularity (Fig. 2a, b, c). Diagnosis of severe aplastic anaemia requires that the patient have at least two of the following: a granulocyte count below 20,000/microlitre and absolute reticulocyte count $<=40 \times 10^{9} / \mathrm{L}$. In addition, the bone marrow biopsy must contain less than $25 \%$. Mild or moderate aplastic anaemia is distinguished from the severe form by the presence of mild or moderate cytopenias and more variable, but still deficient, bone marrow cellularity. These distinctions are more than semantic, they are critical for the prediction of outcome and the choice of therapy. ${ }^{7}$ In the present study, 2 cases were categorized as severe and 4 cases as moderate aplastic anaemias.

In a female child of 14 years with pancytopenia, bone marrow aspiration revealed acute leukemia. On cytochemistry, PAS block positivity and was diagnosed as ALL. The patient was referred to a specialty center for further management (Another case, a male patient of 30 years with pancytopenia, bone marrow aspiration and biopsy revealed lymphoma infiltrating marrow. On immunohistochemistry (IHC), it was diagnosed as T-cell lymphoma. We also encountered a case of primary myelofibrosis, in young male, presenting with weakness, fatigue and gross pallor. Bone marrow aspiration was a dry tap.

Bone marrow trephine biopsy sections revealed myelofibrosis of Grade III, which was more prominent with reticulin stain.

A female patient aged about 22 years was diagnosed with hepatosplenomegaly with pancytopenia. Bone marrow examination revealed numerous sea blue histiocytes with yellow brown pigment diagnosed as Niemann Pick disease.

6 cases showed features of megaloblastic anaemia (fig. 1a, b). Out of the remaining 32 cases, 4 cases, showed dimorphic picture of RBC, a repeat hemogram 2 - 3 weeks after B12, FA and iron replacement therapy showed improvement in counts. The rest of the 28 cases, where fever was the main presenting symptom and PS showed transformed lymphocytes, repeat hemogram done after 15 -20 days interval, showed improvement in the counts.
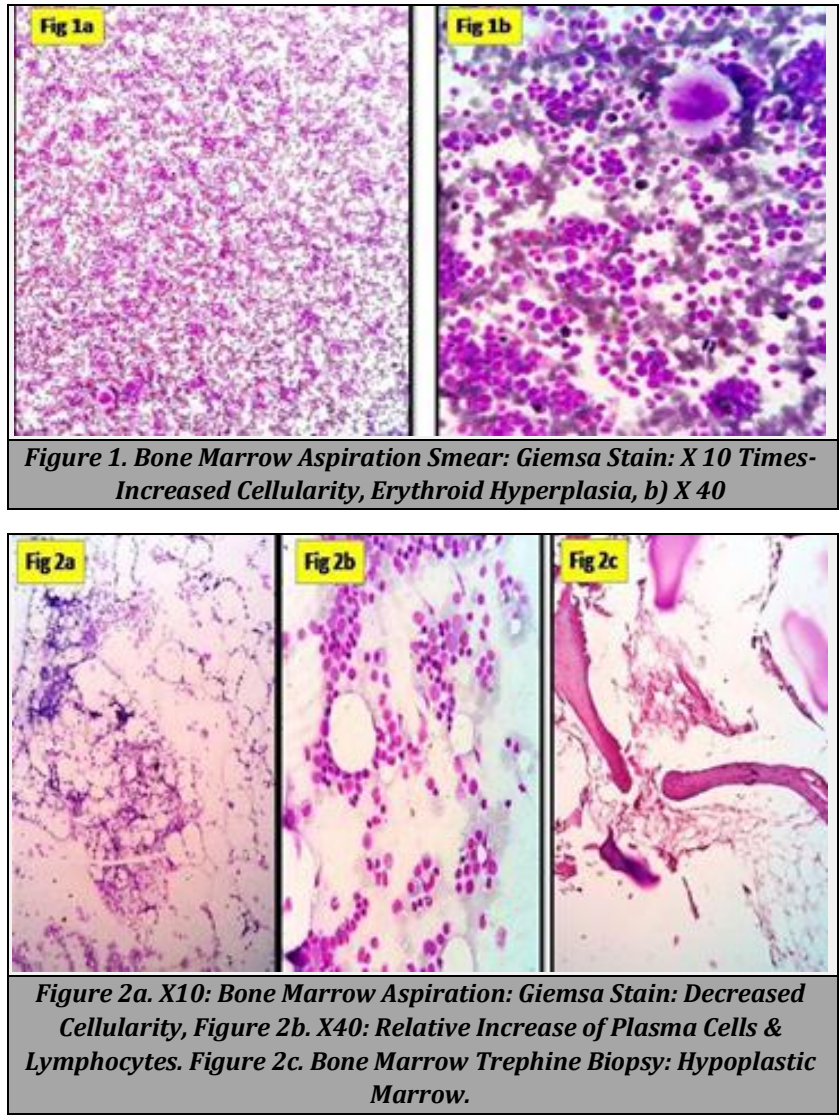
In these 56 cases, only 24 cases (42.85\%)were subjected to bone marrow examination, commonest cause of pancytopenia was episode of viral fever constituting 28 cases (50\%), where the blood counts improved after the fever subsided and when the transformed lymphocytes disappeared from the peripheral blood 4 cases $(7.1 \%)$ showed improvement in counts after vitamin B12 and folic acid replacement therapy. The commonest bone marrow changes were cases of megaloblastic anaemia (10.7\%) and 6 cases of hypoplastic marrow (10.7\%) followed by 2 cases each (3.5\%) of amegakaryocytic thrombocytopenic purpura, acute leukemia (ALL) lymphoma infiltrating marrow $(\mathrm{t}$ cell lymphoma) storage disorder, myelofibrosis, 2 cases of tubercular granuloma, in each of PUO which showed improvement after giving ATT.

\section{CONCLUSIONS}

Though bone marrow examination is an absolute indication in cases of pancytopenia, it is important to wait for at least $2-3$ weeks, do a repeat hemogram, especially in cases of viral fever where the counts usually improve after fever subsides. Also, when dimorphic RBCs are observed in the peripheral blood, a replacement therapy with B12/FA/Iron should be given and if the same picture persists, a bone marrow examination should be done to look for other pathology like MDS etc.

In cases of PUO, bone marrow examination is a very useful investigation. In cases diagnosed as ITP, when the patient does not show improvement in counts, a repeat bone marrow examination should be done, as very rarely; acquired amegakaryocytic thrombocytopenia may be the cause.
Data sharing statement provided by the authors is available with the full text of this article at jemds.com.

Financial or other competing interests: None.

Disclosure forms provided by the authors are available with the full text of this article at jemds.com.

\section{REFERENCES}

[1] Williams WJ, Bentkr E, Erskv AJ. Hematology. 3rd edn. Singapore: McGraw Hill Book Company; 1986:161-84.

[2] Khodke K, Morwah s, Buxi G, et al. Bone marrow examination in case of pancytopenia. J Indian Academy of Clin Med 2001;2:55-59.

[3] Tilak N, Jain R. Pancytopenia--a clinic- hematologic analysis of 77 cases. Indian J Pathol Microbiol 1999;42(4):399-404.

[4] Bodem CR, Hamory BH, Taylor HM, et al. Granulomatous bone marrow disease. A review of the literatureandclinico pathologic analysis of 58 cases. Medicine (Blatimore) 1983;62(6):372-83.

[5] Pease GL. Granulomatous lesions in bone marrow. Blood 1956;11(8):720-34.

[6] Jeevan SKK, Paul-Tara R, Uooin S, et al. Bone marrow granulomas: a retrospective study of 47 cases (A single center experience). American Journal of Internal Medicine 2014;2(5):90-4.

[7] Keisu M, Ost A. Diagnosis in patients with severe pancytopenia suspected of having aplastic anemia. Eur J Hematol 1990;45(1):11-4. 